\title{
Reinventar la clase en la universidad
}

\author{
Mariana Maggio. Buenos Aires: Editorial Paidós, \\ 2018. 183 páginas
}

Jorge Caldera Mercado*

Para nadie puede ser un misterio que la situación mundial y nacional que vivimos ha colocado en cuestionamiento los modos culturales de vivir y de relacionarnos. Nos ha obligado a repensar nuestros modos de interacción y, en especial, en lo que nos convoca en este espacio académico, en aquellos aspectos que permiten avanzar en una educación social y democrática, propia de las exigencias del siglo XXI. Así entendido, es en este contexto descrito que el libro Reinventar la clase en la universidad, de la doctora Mariana Maggio, resulta un aporte necesario para el debate acerca de qué esperamos de la educación y, particularmente, de la educación universitaria. En este sentido, esta publicación aborda el cuestionamiento descrito como una propuesta y desafío para lo que significa la formación educativa universitaria, aportando una perspectiva de análisis crítico respecto de las prácticas docentes presentes en la realidad educativa, realidad en la que el acceso a la información para nuestros estudiantes no solo es fácil, sino que también cada vez más relevante en los requerimientos de comprensión profunda, aspecto que, según la autora, la educación universitaria aún no asimila ni profundiza de manera institucional como parte de sus prácticas docentes, postura que es producto de las didácticas tradicionales que, hoy por hoy, están presentes en las aula universitarias, como modos aún no debatidos abiertamente por la academia. En esencia, esta publicación plantea la pregunta respecto de las prácticas educativas que actualmente son implementadas en las universidades

\footnotetext{
* Chileno. Profesor de Castellano, Universidad Metropolitana de Ciencias de la Educación. Magíster en Innovación Curricular y Evaluación Educativa, Universidad del Desarrollo, Chile. E-mail: jcalderam@gmail.com
} 
y que representan, en sí mismas, un problema para la educación superior, que dificulta a los estudiantes poder acceder y, sobre todo, desarrollar nuevos conocimientos.

Para abordar esta problemática, la autora adopta como método de trabajo, por una parte, la crítica a las prácticas más comunes en las aulas universitarias argentinas y, por otra, la descripción de las prácticas propias implementadas en su propia docencia, y que considera innovadoras, prácticas que son fruto de experiencias investigativas ya descritas en publicaciones anteriores. Con esto, a lo largo de siete capítulos precisa razones y argumentos que permiten comprender la naturaleza y sentido de reinventar la forma en que se realizan las clases universitarias, guiada por una particular pregunta: ¿cuánto tiempo se podrá sostener la educación tradicional sin volverse completamente irrelevante?

Conforme a estos planteamientos iniciales, el libro comienza con el capítulo titulado "La era de la invención", en el que la autora profundiza en diversas preguntas sobre los modos tradicionales de educar en la universidad, destacando, en primer término, cómo estas prácticas están presentes de manera frecuente y sistemática en el hacer de la docencia prácticada por los docentes universitarios. Al respecto, la doctora Maggio sostiene que la docencia, pensada en el formato de la clase propiamente tal, no tiene sentido frente a los nuevos desafíos propuestos por las redes y los medios de comunicación. En este punto pone particular énfasis en la importancia de la creatividad de los docentes para generar en la clase una instancia de aprendizaje que permita inventar, proponer y promover nuevas formas de desarrollar el conocimiento. Para consolidar su idea fuerza, describe la importancia de una "enseñanza poderosa", que permita a los docentes repensar las propias teorías didácticas que implementan en sus clases, así como repensar también las herramientas necesarias para el logro de los aprendizajes. Reconoce, eso sí, que esta labor implica apartarse de los propósitos instaurados por la tradición docente y también la responsabilidad de documentar cada experiencia para así sustentar una nueva teoría asociada a estas innovaciones.

Siguiendo lo descrito, la doctora Maggio agrega otro aspecto involucrado en la problemática y que tiene relación con el escenario 
educativo. Esto lo describe en el segundo capítulo titulado "Tiempos inmersivos", apartado en que analiza este nuevo escenario social y educativo de los estudiantes, en el afán de poner en evidencia qué prácticas culturales están presentes y de qué modo los docentes deben hacer un análisis pedagógico de las mismas. Al respecto, la autora plantea interrogantes específicas y, desde su experiencia personal, utiliza como ejemplo algunas series de televisión, tanto de medios tradicionales como digitales, indagando por aquellos aspectos que nos pueden ser útiles para fomentar un estudio profundo aplicable a la sala de clases. En su opinión, hay mucha didáctica para pensar en las series actuales y, por lo mismo, considera que son importantes porque, fruto de ellas, se puede plantear formas alteradas de realizar la clase, involucrando en esa didáctica preguntas complejas que puedan ayudar a fomentar ejercicios de interpretación desde diferentes planos del conocimiento. Este aspecto, según reconoce, se contituye en un recurso valioso para el desempeño del docente en el aula. Finalmente, reflexiona respecto a cómo dichos aspectos culturales los hacemos protagonistas de nuestras propias propuestas de enseñanza en aula, como recurso para que el tiempo utizado en el análisis fomente el deseo en los estudiantes y el docente por continuar pronto la resolución del enigma propuesto.

Conforme a esta lógica de inmersión, en el tercer capítulo - "Las condiciones que sostienen una clase distinta" - la autora se pregunta por los modos de construcción de las propuestas curriculares presentes en las cátedras universitarias, señalando que su interés no está en cambiar todo, sino más bien está en reenfocar y repensar los modo didácticos utilizados. Como idea fuerza, propone organizar los contenidos más allá de la estructura dada por el programa, focalizando su interés en la comprensión holística que debe tener el docente al momento de abordar el mismo. Para esto, la autora sugiere mirar los programas desde los nodos de conocimiento más que en el orden lineal propuesto, y que generalmente enfatiza la tradición universitaria, logrando con esto que el docente piense en las prácticas, los problemas o los proyectos que fomenten el desarrollo de la creatividad en sus estudiantes. En esencia, la autora plantea como eje central que la práctica docente debe estar centrada en lo que hace el estudiante más que en la explicación dada por el docente, fruto del seguimiento lineal de un programa. Por este mismo aspecto, 
sugiere que el docente, más que enfatizar el programa, debe lograr un abordaje profundo que, en su conjunto, sea una propuesta didáctica en sí misma. La doctora Maggio precisa que lo descrito conlleva como gran problemática pensar y repensar en otros modos de evaluar y otros modos de plantear las bibliografías de un curso. Para la autora, esto tiene sentido si pensamos en la alteración programática como una práctica docente que permita adaptar los espacios y los tiempos de aula como situaciones en constante cambio, fruto del contexto.

En cuanto a la evaluación misma, la doctora Maggio visibiliza esta problemamática en sí misma en el cuarto capítulo titulado "Otra evaluación", enfatizando que la evaluación debe dejar de ser una instancia de comprobación constante de lo que enseñamos. En esta aseveración, la autora precisa que la implementación de didácticas basadas en formas alteradas, tanto en la evaluación como en los otros aspectos descritos en los apartados anteriores, deben ser coherentes con las prácticas creativas que se proponen como formas de enseñanza, prácticas que buscan desarrollar la capacidad crítica de los estudiantes, siempre en la búsqueda de la inclusión de estos en la problemática planteada. La evaluación no puede estar ajena a este requerimiento y, en este sentido, propone una forma de evaluar en diferentes niveles, en la que la coherencia, la creación, la negociación y la inclusión sean elementos orientadores de la práctica docente, ejemplificando nuevamente a partir de su experiencia docente la efectividad que estas prácticas pueden tener. Por último, la autora finaliza este capítulo reflexionando sobre la importancia y necesidad de abordar una discusión acerca de estas nuevos modos de evaluar desde la perspectiva del debate académico, como una forma de apoyar los aprendizajes que los estudiantes desarrollan a partir del fomento de estas prácticas educativas. Enfatiza que no se puede innovar en la didáctica si las prácticas evaluativas siguen siendo las tradicionales.

Integrando los aspectos descritos, en el quinto capítulo - "Una didáctica en vivo" - la autora analiza una perspectiva distinta del problema planteado, enfocando su estudio en las diferentes tendencias culturales y tecnológicas que en la actualidad están presentes, y que son recursos que permiten a los participantes del proceso educativo imaginar. Al respecto, en primer término centra su análisis en los procesos de construcción didáctica de una clase con una didáctica 
en vivo, entendiendo esto último como la realización de clases considerando los elementos culturales y tecnológicos de la actualidad. A continuación, la autora analiza la clase misma como ejercicio posible en las diferentes áreas del conocimiento. Para esto, nuevamente recurre a su experiencia docente para demostrar esta forma de hacer una didáctica en vivo, ligando estos aspectos a los enunciados en el primer capítulo, en especial respecto de las interrogantes referidas a las formas de plantear las clases y a los abordajes didácticos, todo esto considerando la mirada inventiva e inmersiva propuesta. En particular, se pregunta por la fundamentación de las decisiones que sustentan sus planteamientos y cómo estos ayudan a la labor que significa intervenir en los medios y los modos de desarrollar aprendizajes. En cualquier caso, sostiene que las ventanas que se abren con estas prácticas permiten nuevas formas de hacer didáctica y permiten al docente, a su vez, situarse en escenarios creativos no abordados. Como último aspecto, reflexiona sobre el aporte significativo que tiene proponer clases "desde afuera" (considerando lo cultural y lo tecnológico) y cómo la utilización de estos recursos ayuda al proceso educativo de todos.

En el sexto capítulo titulado "Las clases como una experiencia que hay que vivir", la autora aborda la realidad de las universidades argentinas enfatizando cómo su rol público involucra aspectos que permiten el acceso democrático al conocimiento. Sin embargo, también destaca que en dichas instituciones no se están logrando los fines descritos. Para esto enuncia un conjunto de observaciones de la realidad universitaria argentina que describen cómo la deserción temprana se presenta de manera frecuente en las instituciones. Frente a este escenario, la doctora Maggio propone que la misión de la docencia debe estar en reenfocarse en su quehacer en el aula. Así, la didáctica de los docentes debe constituirse en una experiencia que contribuya a la vida de los estudiantes. ¿Cómo lograr esto? En primer término, sostiene que el acceso a los recursos está disponible y en fuentes diversas son aspectos que los estudiantes tienen de manera distinta al aula. Afirma que se deben aprovechar esos recursos y, además, diseñar clases que permitan debatir problematicas que interesen y pongan en acción la inventiva y las interacciones de los estudiantes con sus pares, aspecto que, sin duda, fomenta la divergencia y la creatividad. Este aspecto lo refuerza al finalizar este capítulo, señalando que la experiencia de 
la clase bajo este enfoque es algo que vale la pena vivir, tanto para el docente como para el estudiante, y sostiene que es, en esencia, un compromiso político que involucra la decisión de educar para todos los que realizamos docencia.

Como una forma de conclusión, en el último capítulo, "La enseñanza como proyecto colectivo", la autora analiza su diálogo con docentes, a partir de su experiencia participando en reuniones de colaboración de la misma asignatura, instancia que valora y en la que pudo discutir el diseño de propuestas para las clases y las evaluaciones, y en la que, además, fue posible compartir ideas, materiales, propuestas y experiencias, aspectos que desarrollan en los docentes lo que denomina "inteligencia colectiva", aspecto que Maggio identifica como una marca de época. ¿Qué depara para el futuro educativo según esta autora? Sin duda, la necesidad de abrir los espacios de la asignatura a los estudiantes, mejorar los diseños de las clases y también los procesos evaluativos involucrados en los mismos. Conforme a esto, la autora concluye que la labor universitaria está en llevar adelante iniciativas que permitan formar a docentes con un espíritu de trasformación, siempre con el interés en aportar a los jóvenes para que puedan cumplir sus sueños, sus expectativas y crear, con esto, un mundo más justo.

Esta obra permite abordar una problemática latente en la educación superior, cual es la forma en que se realiza la docencia universitaria y cómo ésta se configura desde lo tradicional, mostrando fundamentaciones interesantes que ayudan a comprender la problemática analizada y la importancia de implementar nuevas formas creativas de hacer la docencia, que enriquezcan las aulas universitarias con nuevos enfoques didácticos y fomenten un rol activo por parte de los estudiantes; ello permitirá un aprendizaje profundo y comprensivo de las temáticas analizadas en el aula. Este libro es, sin duda, recomendable para aquellos que se interesan por conocer pasos y secuencias que le permitan crear y visualizar propuestas de cambio en sus aulas. La principal virtud de este libro es la sinceridad para mostrar, desde las preguntas y descripción de experiencias docentes, aquellos aspectos que la motivan a ser una profesional formadora, capaz de motivar a sus estudiantes desde lo cotidiano, aspecto que promueve como actitud para aquellos que lean este libro. 